\title{
Planification des besoins selon l'art. 55a LAMal: position de la FMH
}

\section{Situation initiale}

1. La planification des besoins selon l'art. 55a LAMal sera introduite par voie d'ordonnance et ne peut désormais être remise en question ni politiquement ni juridiquement. Cela vaut en particulier sur le plan politique, où toute intervention se heurterait à un front commun - si rare - entre le DFI et les directeurs cantonaux des affaires sanitaires.

2. L'ordonnance est en outre grevée d'une multitude de problèmes de méthode et de mode d'opération non résolus. Or, vu l'intention du DFI et des directeurs cantonaux de la santé de l'introduire au plus tôt, ces questions ne pourront plus faire l'objet d'une clarification.

3. L'objection majeure est et demeure cependant que l'ordonnance portera un préjudice sérieux et durable aux jeunes médecins, avec en plus le risque d'un vieillissement du corps médical. Cette objection a d'autant plus de poids qu'il n'a jamais été démontré que cette limitation de l'admission à pratiquer à la charge de l'assurance-maladie pouvait vraiment atteindre son but, à savoir un ralentissement de la croissance des coûts.

\section{Exigences}

Malgré ces importantes réserves, la FMH est prête à collaborer à la mise en application de ces dispositions, toutefois aux conditions suivantes:

a. La durée de validité de l'ordonnance ne doit pas s'étendre au-delà du 31 décembre 2003 au plus tard ou est fixée à 18 mois depuis l'entrée en vigueur.

b. Ce laps de temps est utilisé pour élaborer une planification des besoins qui soit souple, réponde aux intérêts médicaux de notre population et prenne en compte tous les fournisseurs de prestations de manière égale.

c. La Confédération prend immédiatement les mesures nécessaires pour l'élaboration d'une liste positive indiquant les prestations prises en charge par l'assurance obligatoire des soins.

d. Le législateur renonce à une modification de l'obligation de contracter et encourage à recourir en premier lieu à toutes les possibilités offertes par la loi.

La Fédération des médecins suisses (FMH) est consciente du fait que l'ouverture possible de 300 cabinets médicaux supplémentaires par des médecins étrangers déjà établis en Suisse aboutirait à une augmentation des coûts considérable et non justifiée sur le plan médical; c'est pourquoi elle accepte l'introduction de l'ordonnance comme mesure d'urgence, sous réserve des conditions susmentionnées. Elle sait aussi qu'elle exige ainsi de ses jeunes membres un lourd tribut; qu'ils sachent cependant que, si les conditions résumées ci-dessus ne sont pas remplies, elle les soutiendra pleinement dans les actions de lutte qu'ils entreprendront. 